\title{
Research on Poisson Point Process Model of Transmission System and Its Reliability
}

\author{
Yonghua Zhu, Chenglin Chen and Tian Zhang \\ Department of Mathematics and Physics, North China Electric Power University, Beijing, China
}

\begin{abstract}
This paper mainly discussed the system reliability with the new isolated circuit breaker and the traditional equipment in operation process. Firstly, we established the Poisson marked point process model of the outage time and derived their mathematical expectations. And we used the expectations as the index to evaluate the reliability of transmission system. Then we used the censored data to get the Bayesian estimation of the parameters. Finally we decomposed the system outage time to calculate the result of the reliability model. By the analysis, we provided a reference for the reliability evaluation of transmission system.
\end{abstract}

Keywords-poisson marked point process; bayesian estimation; outage time

\section{INTRODUCTION}

The new generation of intelligent substation is a major technological innovation of State Grid Corporation of China since 2012. One of its key technologies is the isolated circuit breaker which is equal to the function of the traditional circuit breaker and isolating switch. Its characters are complex manufacturing processes, high price and less maintenance requirement. In a system, because of the lower reliability of the traditional circuit breakers, we usually put the isolating switches on the both ends of them to ensure the safety of maintenance personnel and other equipment. For the technology is increasing mature, its failure rates are less and less. While the failures of the isolating switches are no decrease. In this circumstance, State Grid launched the new isolated circuit breaker. Here we mainly consider whether the substation need the isolated circuit breakers to replace the traditional equipment, and investigate its influences on the reliability of power supply system.

\section{Reliability Model of Single Parent Segment SYSTEM}

Due to the equivalent structure of the model, we just investigate one side. The model is divided into two parts: one part is the single bus system on a certain side (a bus connect a main variable interval and four outgoing line intervals), the other part is the middle isolation part (a system connect the circuit breakers of the two single bus systems and the isolating switches on the both side). When there are failures in a main variable interval, the middle part will be connected and the power supply at the other end feed rapidly the users of the fault side to reduce the outage time. So this time will be deducted from the total bus outage time.

\section{A. Modeling Assumption}

In the time interval $(0, t]$, we record the length $L$ of system outage time during the maintenance, and mark them on the timeline according to the sequence. Then we get a compound Poisson process of marked point $L$. We assume that the number of outage resulted by system failures is a homogeneous Poisson process $\{N(t), t \geq 0\}$ of intensity $\lambda$. There is a marked point $L$ in the point event, in which $L$ is a random variable and its probability density function is $f(l)$. When calculate the system outage time, in the neighborhood $\Delta t$ of a certain time $t_{\alpha}$, we assume the probability is $o(\Delta t)$ when the two mutual independent equipment break down at the same time, namely the probability is 0 . Because the maintenance time is far less than the system uptime, it is just as a marked point of the point events rather than the research time interval. The new isolated circuit breaker is equivalent to the traditional circuit breaker and current transformer in series. We assume the two equipment are independent of each other, and their failure times and maintenance time are independent random variables.

\section{B. Traditional Equipment Condition}

The single parent segment system under the traditional equipment condition is as follows:

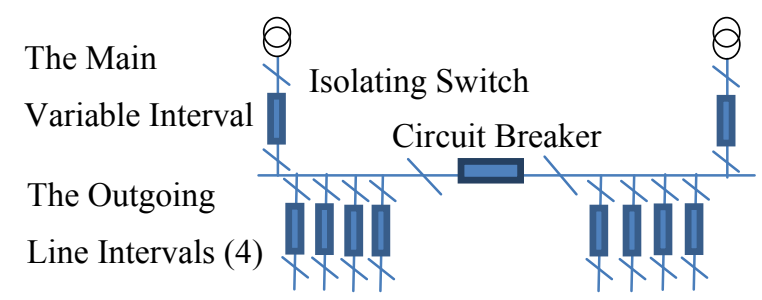

FIGURE I. THE SINGLE PARENT SEGMENT SYSTEM UNDER THE TRADITIONAL EQUIPMENT CONDITION.

We assume $\left\{N_{A}(t), t \geq 0\right\}$ is a homogeneous Poisson process of intensity $\lambda_{A}$ and $N_{A}(t)$ represents the failure times of circuit breakers in the time interval $(0, t]$. Similarly, $\left\{N_{B 1}(t), t \geq 0\right\}$ and $\left\{N_{B 2}(t), t \geq 0\right\}$ represents respectively the failure times of the isolating switches on each end in $(0, t]$, and they are independent and have same intensity $\lambda_{B}$. Then we assume $\left\{N_{i}(t), t \geq 0\right\} \quad(i=1,2,3,4)$ is a homogeneous Poisson process of intensity $\lambda_{i}$, and it represents the failure times of the ith outgoing line interval in $(0, t]$. In the time interval $(0, t]$, the failure times of outgoing line interval is equal to the sum 
of the failure times of the three equipment, that is: $N_{i}(t)=N_{A}(t)+N_{B 1}(t)+N_{B 2}(t)$. Base on the superposition theorem of homogeneous Poisson process, we get that: $\lambda_{i}=\lambda_{A}+2 \lambda_{B}$.

For the point events of each failure, we use the corresponding marked points to represent the total bus outage time resulted by each failure. We assume that $L_{X P}(n)$ is the bus outage time and its probability density function is $f_{x p}(l)$. We construct the following compound Poisson process:

$$
T_{i}(t)=\sum_{n_{i}=1}^{N_{i}(t)} L_{X P}\left(n_{i}\right)
$$

$T_{i}(t)$ is the total bus outage time resulted by the failures of the ith outgoing line interval in $(0, t]$.

Similarly, we assume $\left\{N_{0}(t), t \geq 0\right\}$ is a homogeneous Poisson process of intensity $\lambda_{0}$ and $N_{0}(t)$ is the failure times in the main variable interval in $(0, t]$. Then we get: $\lambda_{0}=\lambda_{A}+2 \lambda_{B}$.

It is assumed that $L_{X}(n)$ is the outage time of the main variable interval resulted by the nth failure and its probability density function is $f_{x}(l)$. Then we construct the compound Poisson process of the outage time in the main variable interval:

$$
T_{0}(t)=\sum_{n_{0}=1}^{N_{0}(t)} L_{X}\left(n_{0}\right)
$$

Here $L_{X}(n)$ and $L_{X P}(n)$ are two different random variables. $L_{X P}(n)$ is the bus outage time in the outgoing line interval that just involves the situation resulted by the failures of isolating switches near the bus. While $L_{X}(n)$ is the bus outage time in the main variable interval, we need to consider the system outage time resulted by the failures of the three equipment at the same time.

To the middle isolation part, in the practical work, it may not be closed as a result of equipment failures, or the system cannot be connected at both ends by the operating troubles. We assume the probability that it cannot be connected is $p_{0}$ when there are failures in the middle isolation part. Besides, the probability is $p_{x p}^{\prime}$ when there are failures in the main variable interval but the bus does not outage. We define $\left\{N_{0}^{\prime}(t), t \geq 0\right\}$ is a homogeneous Poisson process of intensity $\lambda_{0}^{\prime}$, and $N_{0}^{\prime}(t)$ represents the times that the bus does not outage when there are failures in the main variable interval in $(0, t]$. Base on the sparse theorem, we can get:

$$
\lambda_{0}^{\prime}=\left(1-p_{0}\right) p_{x p}^{\prime}\left(\lambda_{A}+2 \lambda_{B}\right)
$$

We use the marked point $L_{0}(n)$ to represent the outage time of the main variable interval resulted by each failure, in which it obeys the probability density function $f_{x^{\prime}}(l)$. $\left\{T_{p}(t), t \geq 0\right\}$ represents the compound Poisson process of the total bus outage time. Then the process can be decomposed into:

$$
\begin{aligned}
& T_{p}(t)=\sum_{n_{1}=1}^{N_{1}(t)} L_{X P}\left(n_{1}\right)+\sum_{n_{2}=1}^{N_{2}(t)} L_{X P}\left(n_{2}\right)+\sum_{n_{3}=1}^{N_{3}(t)} L_{X P}\left(n_{3}\right) \\
& +\sum_{n_{4}=1}^{N_{4}(t)} L_{X P}\left(n_{4}\right)+\sum_{n_{0}=1}^{N_{0}(t)} L_{X}\left(n_{0}\right)-\sum_{n_{0}^{\prime}=1}^{N_{0}^{\prime}(t)} L_{0}\left(n_{0}^{\prime}\right) .
\end{aligned}
$$

In this case, the overall average time of bus outage is:

$$
E\left[T_{p}(t)\right]=\left(\lambda_{A}+2 \lambda_{B}\right) t\left[E\left(L_{X}\right)+4 E\left(L_{X P}\right)-\left(1-p_{0}\right) p_{x p}^{\prime} E\left(L_{0}\right)\right] .
$$

\section{New Equipment Condition}

The following schematic is the single parent segment system under the new equipment condition.

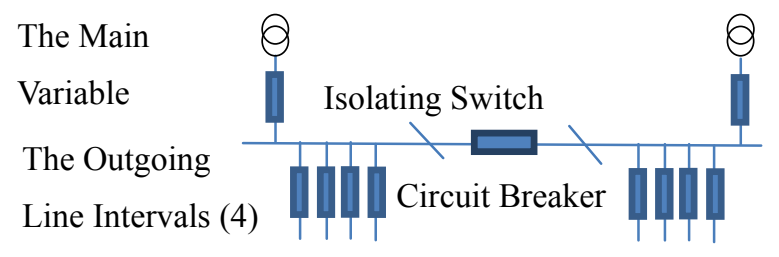

FIGURE II. THE SINGLE PARENT SEGMENT SYSTEM UNDER THE NEW EQUIPMENT CONDITION.

In this system, there is no isolating switch at both ends of the isolated circuit breakers. For the less service time, the failure data is few. Because of the structure and working principle, the new isolated circuit breaker is equivalent to cascade the circuit breaker and current transformer.

Same to the traditional condition in the above, $\left\{N_{A}(t), t \geq 0\right\},\left\{N_{i}(t), t \geq 0\right\}(i=1,2,3,4)$ and $\left\{N_{0}(t), t \geq 0\right\}$ also represent those failure times. $\left\{N_{C}(t), t \geq 0\right\}$ represents the failure times of the current transformers in $(0, t]$ and its intensity is $\lambda_{C}$. For the superposition theorem, we get that: $\lambda_{i}=\lambda_{A}+\lambda_{C}, \lambda_{0}=\lambda_{A}+\lambda_{C}$.

On the basis of these Poisson processes,

$$
\begin{aligned}
& T_{i}(t)=\sum_{n_{i}=1}^{N_{i}(t)} L_{Y P}\left(n_{i}\right) . \\
& T_{0}(t)=\sum_{n_{0}=1}^{N_{0}(t)} L_{Y}\left(n_{0}\right) .
\end{aligned}
$$


Thereinto we assume $L_{Y P}(n)$ is the bus outage time resulted by the nth failure and $L_{Y}(n)$ is the outage time in the main variable interval resulted by the nth failure.

Different from the traditional type, only if there are failures in the low voltage cell of the current transformers in the main variable interval, the bus can be electrified and the middle isolation part can be closed during the maintenance. Here we assume the probability is $p_{y p}^{\prime}$ when there are failures in the main variable interval but the bus does not outage. Same to $\lambda_{0}^{\prime}$ and $N_{0}^{\prime}(t)$ in the previous section, we get that:

$$
\lambda_{0}^{\prime}=\left(1-p_{0}\right) p_{y p}^{\prime}\left(\lambda_{A}+\lambda_{C}\right)
$$

Similarly, the marked point $L_{0}(n)$ represents the outage time of the main variable interval resulted by each failure, in which its probability density function is $f_{y^{\prime}}(l)$. For the compound Poisson process $\left\{T_{p}(t), t \geq 0\right\}$ of the total bus outage time,

$$
\begin{aligned}
& T_{p}(t)=\sum_{n_{a}=1}^{N_{a}(t)} L_{Y P}\left(n_{a}\right)+\sum_{n_{b}=1}^{N_{b}(t)} L_{Y P}\left(n_{b}\right)+\sum_{n_{c}=1}^{N_{c}(t)} L_{Y P}\left(n_{c}\right) \\
& +\sum_{n_{d}=1}^{N_{d}(t)} L_{Y P}\left(n_{d}\right)+\sum_{n_{0}=1}^{N_{0}(t)} L_{Y}\left(n_{0}\right)-\sum_{n_{0}^{\prime}=1}^{N_{0}^{\prime}(t)} L_{0}\left(n_{0}^{\prime}\right) .
\end{aligned}
$$

So the overall average time of bus outage is:

$$
E\left[T_{p}(t)\right]=\left(\lambda_{A}+\lambda_{C}\right) t\left[E\left(L_{Y}\right)+4 E\left(L_{Y P}\right)-\left(1-p_{0}\right) p_{y p}^{\prime} E\left(L_{0}\right)\right] .
$$

\section{The Calculation of Reliability Model}

\section{A. Parameter Estimation of the Model}

If the intensity of a homogeneous Poisson process is $\lambda$, then the number of point events in $(0, t]$ obeys the Poisson distribution with parameter $\lambda t$, and the time interval of two successive point events obeys the exponential distribution with parameter $\lambda$. Therefore, we adopt the censored data in a fixed time interval to estimate the intensity $\lambda$ of the homogeneous Poisson process.

In the time interval $(0, T]$, we observe same n-equipment that their lives are independent and identical distribution, and obey the exponential distribution with parameter $\lambda$. When stop observing, there are failures in r-equipment, in which the fault time is $0<t_{1} \leq t_{2} \leq \cdots \leq t_{r} \leq T$, then the likelihood function is determined:

$$
L\left(t_{1}, t_{2}, \ldots, t_{r} \mid \lambda\right)=C \lambda^{r} e^{-\lambda S} .
$$

Among this formula, $C=n ! /(n-r) !, S=\sum_{i=1}^{r} t_{i}+(n-r) T$. If there is no prior information of equipment, we can use the non-informative prior distribution of $\lambda$ as the prior information, and determine the quadratic loss function. Then the Bayesian estimation of $\lambda$ is:

$$
\hat{\lambda}=\int_{0}^{\infty} \lambda \pi(\lambda \mid r) d \lambda=r / S .
$$

We adopt their censored data in a fixed time interval. After data filter and analysis, we use the failure data of the equipment under the $220 \mathbf{K V}$ voltage in a certain substation of a Chinese province. There are 14 circuit breakers, 48 isolating switches and 42 current transformers. The corresponding data are listed in the following tables:

TABLE I. THE CENSORED DATA OF CIRCUIT BREAKERS OPERATING TIME (UNIT: YEAR)

\begin{tabular}{|l|l|l|l|l|l|l|}
\hline \multicolumn{7}{|c|}{ Operating Time } \\
\hline 3.92896 & 5 & 5 & 5 & 5 & 5 & 5 \\
\hline 4.75205 & 5 & 5 & 5 & 5 & 5 & 5 \\
\hline
\end{tabular}

From the above Table I, the fixed time interval is 5 years, during the observation, the failure times: $r=2, S=68.68101$. Due to the lack of the prior information, we adopt the noninformative prior distribution. Then the Bayesian estimation of $\lambda_{A}$ is: $\hat{\lambda}_{A}=r / S=0.02912$.

Similarly, we can determine the Bayesian estimation of $\lambda_{B}$ and $\lambda_{C}$ :

TABLE II. THE CENSORED DATA OF ISOLATING SWITCHES OPERATING TIME (UNIT: YEAR)

\begin{tabular}{|c|r|r|r|r|r|r|r|}
\hline \multicolumn{8}{|c|}{ Operating Time } \\
\hline 0.54918 & 2 & 2 & 2 & 2 & 2 & 2 & 2 \\
\hline 1.44932 & 2 & 2 & 2 & 2 & 2 & 2 & 2 \\
\hline 2 & 2 & 2 & 2 & 2 & 2 & 2 & 2 \\
\hline 2 & 2 & 2 & 2 & 2 & 2 & 2 & 2 \\
\hline 2 & 2 & 2 & 2 & 2 & 2 & 2 & 2 \\
\hline 2 & 2 & 2 & 2 & 2 & 2 & 2 & 2 \\
\hline
\end{tabular}

From the Table II, the fixed time interval is 2 years, $r=2$, $S=93.9985$. Then $\hat{\lambda}_{B}=r / S=0.02128$.

TABLE III. THE CENSORED DATA OF CURRENT TRANSFORMERS OPERATING TIME (UNIT: YEAR)

\begin{tabular}{|l|l|l|l|l|l|l|}
\hline \multicolumn{7}{|c|}{ Operating Time } \\
\hline 1.52329 & 2 & 2 & 2 & 2 & 2 & 2 \\
\hline 1.94247 & 2 & 2 & 2 & 2 & 2 & 2 \\
\hline 1.81096 & 2 & 2 & 2 & 2 & 2 & 2 \\
\hline 1.52055 & 2 & 2 & 2 & 2 & 2 & 2 \\
\hline 1.24384 & 2 & 2 & 2 & 2 & 2 & 2 \\
\hline 1.80822 & 2 & 2 & 2 & 2 & 2 & 2 \\
\hline
\end{tabular}

From the Table III, the fixed time interval is 2 years, $r=2$, $S=81.84933$. Then $\hat{\lambda}_{C}=r / S=0.07331$. 


\section{B. Single Parent Segment System under the Traditional Equipment Condition}

We can calculate the mean time to failure of circuit breakers and isolating switches directly by the statistical data (unit: hour): $E\left(L_{A}\right)=23.78382, E\left(L_{B}\right)=14.90096$.

So we get the mean time of single maintenance in the main variable interval:

$$
E\left(L_{X}\right)=\frac{\lambda_{A} E\left(L_{A}\right)}{\lambda_{A}+2 \lambda_{B}}+\frac{2 \lambda_{B} E\left(L_{B}\right)}{\lambda_{A}+2 \lambda_{B}}=18.50963 .
$$

We redefine that $\left\{N_{P}(t), t \geq 0\right\}$ is a homogeneous Poisson process of intensity $\lambda_{P}$ and $N_{P}(t)$ represents the failure times of the bus in $(0, t]$, that is the failure times of the bus resulted by the failures of the isolating switches close to the bus. The above failures include the own running failures and the operating troubles resulted by the maintenance of circuit breakers and the isolating switches far from the bus. The intensity is $\lambda_{B}$ in the Poisson process of the running failures. And the intensity of Poisson process of the operating troubles can be derived from the intensity of Poisson process of the failures of the circuit breakers the isolating switches close to the bus in a certain probability $p_{x}$ by sparse theory, that is $p_{x}\left(\lambda_{A}+\lambda_{B}\right)$. Besides, as the structural features of isolating switch, the failures of the isolating switches close to the bus does not necessarily result in the bus outage. The intensity of the Poisson process of the bus outage can be got from a probability $p_{B}$ by sparse theory, that is:

$$
\lambda_{P}=p_{B}\left[\lambda_{B}+p_{x}\left(\lambda_{A}+\lambda_{B}\right)\right]
$$

For the expert experience, $p_{x}=0.6, p_{B}=0.5$, so we get: $\lambda_{P}=0.02576$.

Based on the fundamental point process $\left\{N_{P}(t), t \geq 0\right\}$, we use the marked point $L_{B}$ of the failure time of the isolating switches close to the bus. So we can get:

$$
T_{B}(t)=\sum_{n=1}^{N_{P}(t)} L_{B}(n)
$$

Then

$E\left[T_{p}(t)\right]=E\left[T_{B}(t)\right]=\lambda_{P} t E\left(L_{B}\right)=0.38385 t=\left(\lambda_{A}+2 \lambda_{B}\right) t E\left(L_{X P}\right)$.

So we get the overall average time of bus outage in $(0, t]$.

We redefine that $\left\{N_{0}^{\prime}(t), t \geq 0\right\}$ is a homogeneous Poisson process of intensity $\lambda_{0}$, in which $N_{0}^{\prime}(t)$ represents the times that there are failures in the main variable interval but the bus does not outage in the time interval $(0, t]$, and there is:

$$
\lambda_{0}^{\prime}=\left(1-p_{0}\right) p_{x p}^{\prime}\left(\lambda_{A}+2 \lambda_{B}\right)
$$

Thereinto, $p_{0}$ represents the probability that it cannot be connected when the middle isolation part breaks down. And $p_{x p}^{\prime}$ represents the probability that there are failures in the main variable interval but the bus does not outage. Based on the expert experience, $p_{0}=0.01$.

Actually, the bus outage time is only related to the isolating switches close to the bus. So the overall average time of bus outage can be deduced by the expectations of the compound Poisson process of the failures of the isolating switches close to the bus. The intensity of the Poisson process of failures in a main variable interval is: $\lambda=\lambda_{A}+2 \lambda_{B}=0.07186$.

Then the probability is: $\lambda_{P} /\left(\lambda_{A}+2 \lambda_{B}\right)=0.359375$ when there are failures in the main variable interval and the bus outage, so $p_{x p}^{\prime}=1-\lambda_{P} /\left(\lambda_{A}+2 \lambda_{B}\right)=0.64063$.

When there are failures in the main variable interval but the bus does not outage, $E\left(L_{0}\right)$ is the expectation of maintenance time $L_{0}$ in the main variable interval. In $(0, t]$, we define that $T(t), T_{p}(t), \bar{T}_{p}(t)$ are the outage time, the bus outage time and the time that the bus does not outage when there are failures in the main variable interval, then

$$
T(t)=T_{p}(t)+\bar{T}_{p}(t), E\left[\bar{T}_{p}(t)\right]=E[T(t)]-E\left[T_{p}(t)\right] .
$$

$\left(\lambda_{A}+2 \lambda_{B}\right) t E\left(L_{0}\right)=\left(\lambda_{A}+2 \lambda_{B}\right) t E\left(L_{X}\right)-\left(\lambda_{A}+2 \lambda_{B}\right) t E\left(L_{X P}\right)$ $=0.94292 t$.

In this case, the overall average time of bus outage is:

$$
\begin{aligned}
& E\left[T_{p}(t)\right]=\left(\lambda_{A}+2 \lambda_{B}\right) t E\left(L_{X}\right)+4\left(\lambda_{A}+2 \lambda_{B}\right) t E\left(L_{X P}\right) \\
& -\left(1-p_{0}\right) p_{x p}^{\prime}\left(\lambda_{A}+2 \lambda_{B}\right) t E\left(L_{0}\right)=2.26415 t .
\end{aligned}
$$

\section{Single Parent Segment System under the New Equipment Condition}

Similarly, the mean time to failure of the current transformers is: $E\left(L_{C}\right)=10.59051$.

We get the mean time of a single maintenance in the outgoing line interval:

$$
E\left(L_{Y}\right)=\frac{\lambda_{A} E\left(L_{A}\right)}{\lambda_{A}+\lambda_{C}}+\frac{\lambda_{C} E\left(L_{C}\right)}{\lambda_{A}+\lambda_{C}}=14.34126 .
$$


When there are failures in the low voltage cell of the current transformers but the bus does not outage, through a certain probability $p_{C}$, the Poisson process of the times that the bus outage can be derived from the Poisson process of failure times of the current transformers on account of the sparse theory. Obviously, the intensity of the Poisson process is $p_{C} \lambda_{C}$. According to expert experience and some information, $p_{C}=0.6, E\left(L_{Y P}\right)=\frac{\lambda_{A} E\left(L_{A}\right)}{\lambda_{A}+\lambda_{C}}+\frac{p_{C} \lambda_{C} E\left(L_{C}\right)}{\lambda_{A}+\lambda_{C}}=11.30937$.

We define that $\left\{N_{0}^{\prime}(t), t \geq 0\right\}$ is a homogeneous Poisson process of intensity $\lambda_{0}^{\prime}$ and it represents the times that the bus does not outage when there are failures in the main variable interval in $(0, t]$. And there is:

$$
\lambda_{0}^{\prime}=\left(1-p_{0}\right) p_{y p}^{\prime}\left(\lambda_{A}+\lambda_{C}\right)
$$

Similarly, $p_{0}=0.01 \cdot p_{y p}^{\prime}$ is the probability that the bus does not outage when there are failures in the main variable interval. And then we get the intensity of Poisson process of the bus outage resulted by the failures of circuit breakers in the outgoing line interval, $\lambda_{P}=\lambda_{A}+p_{C} \lambda_{C}=0.07311$.

Then the probability is: $\lambda_{P} /\left(\lambda_{A}+\lambda_{C}\right)=0.71372$ when there are failures in the outgoing line interval and the bus outage. Thanks to the same situation between the main variable interval and the outgoing line interval, $p_{y p}^{\prime}=1-\lambda_{P} /\left(\lambda_{A}+\lambda_{C}\right)=0.28628$.

Same as the traditional condition, to get $E\left(L_{0}\right)$, define $T(t), T_{p}(t), \bar{T}_{p}(t)$, then we also get (18) and

$$
\begin{aligned}
& \left(\lambda_{A}+\lambda_{C}\right) t E\left(L_{0}\right)=\left(\lambda_{A}+\lambda_{C}\right) t E\left(L_{Y}\right)-\left(\lambda_{A}+\lambda_{C}\right) t E\left(L_{Y P}\right) \\
& =0.31057 t .
\end{aligned}
$$

Finally we get the overall average time of bus outage:

$$
\begin{aligned}
& E\left[T_{p}(t)\right]=\left(\lambda_{A}+\lambda_{C}\right) t E\left(L_{Y}\right)+4\left(\lambda_{A}+\lambda_{C}\right) t E\left(L_{Y P}\right) \\
& -\left(1-p_{0}\right) p_{y p}^{\prime}\left(\lambda_{A}+\lambda_{C}\right) t E\left(L_{0}\right)=6.0146 t .
\end{aligned}
$$

From the Table IV, by comparing the failure rates, as the equipment in power supply system, the reliability of the three kinds of equipment is very high. If we only consider about them, for the influence of on the system reliability, the current transformer is bigger than circuit breaker and isolating switche.

When observing the MTTR, we find the ones of circuit breakers are more than the isolating switches and current transformers. Because of the more MTTR, it is concluded that circuit breaker has more complex structure and more difficult maintenance. Therefore, if just consider about a single maintenance time, the circuit breakers have the bigger influence on system outage time for equipment failures than the isolating switches and current transformers.

\section{B. System Reliability}

TABLE V. THE INDEX OF SYSTEM RELIABILITY

\begin{tabular}{|c|c|c|c|}
\hline Reliability Index & $\begin{array}{c}\text { Failure } \\
\text { rate } \lambda\end{array}$ & $\begin{array}{c}\text { The overall } \\
\text { average time } \\
\text { of bus } \\
\text { outage } \\
E\left[T_{p}(t)\right]\end{array}$ & $\begin{array}{c}\text { The overall } \\
\text { average outage } \\
\text { time of } \\
\text { outgoing line } \\
\text { interval } \\
E[T(t)]\end{array}$ \\
\hline Traditional equipment & 0.3584 & $2.26415 t$ & $9.37362 t$ \\
\hline New equipment & 0.51215 & $6.0146 t$ & $14.87499 t$ \\
\hline
\end{tabular}

From the Table V, through the comparison of the system reliability, in the single parent segment system, the reliability under the traditional equipment condition is better than the system reliability under traditional equipment conditions. So we suggest that the traditional equipment should be kept rather than the new isolated circuit breakers in the above transmission system.

\section{REFERENCES}

[1] Deng Yonglu, Liang Shunzhi, Stochastic Point Process and its Application, Science Press, Beijing, 1992.

[2] Neyman J., Scott E.L., A Statistics Approach to Problem of Cosmology, Journal of the Royal Statistical Society, 1958, pp. 1-43.

[3] Wold H., On Stationary Point Processes and Markov Chains, Skand. Aktuar., 1948, pp. 229-240.

[4] Khinchin A.Ya., On Poisson Streams of Events, Theor. Prob. Appl., 1956, pp. 248-255.

[5] Snyder D. L., Random Point Processes, Wiley, New York, 1975.

\section{COMPARISON AND ANALYSIS}

\section{A. Equipment Reliability}

TABLE IV. THE INDEX OF EQUIPMENT RELIABILITY

\begin{tabular}{|c|c|c|c|}
\hline Reliability Index & $\begin{array}{c}\text { Failure } \\
\text { rate } \lambda\end{array}$ & $\begin{array}{c}\text { Average } \\
\text { lifetime } \lambda^{-1}\end{array}$ & $\begin{array}{c}\text { Mean time to } \\
\text { repair (MTTR) } \\
E(L)\end{array}$ \\
\hline Circuit breaker & 0.02912 & 34.34 & 23.78382 \\
\hline Isolating switch & 0.02128 & 46.99 & 14.90096 \\
\hline Current transformer & 0.07331 & 13.64 & 10.59051 \\
\hline
\end{tabular}

